\title{
Prediction of Maximum Dry Density and Unconfined Compressive Strength of Cement Stabilised Soil Using Artificial Intelligence Techniques
}

\author{
Shakti Suman $^{1} \cdot$ Mahasakti Mahamaya $^{1} \cdot$ Sarat Kumar Das $^{1}$
}

Received: 20 February 2016/ Accepted: 21 March 2016/Published online: 8 April 2016

(C) Springer International Publishing Switzerland 2016

\begin{abstract}
The soft soil that has not enough in situ bearing capacity needs proper stabilization before any construction can be done on this soil. Cement stabilization has been found to be an effective method to improve the soil properties by many researchers. The strength development in a cement stabilized mix depends on a number of factors such as the soil properties, the water-cement ratio and the percentage of cement in the mix. In the present study an attempt is made to develop prediction model to determine the maximum dry density (MDD) and the unconfined compressive strength (UCS) of cement stabilized soil with the use of two recently developed artificial intelligence (AI) techniques; functional networks (FN) and multivariate adaptive regression splines (MARS). Database previously available in the literature was used to develop the prediction models. Based on different statistical performance criteria, it was found that the FN and MARS techniques, are better at prediction of MDD and UCS as compared to previously used AI techniques, artificial neural network and support vector machine. The prediction model presented here is more comprehensive and can be used by professional engineers.
\end{abstract}

Sarat Kumar Das

saratdas@rediffmail.com

Shakti Suman

shaktisuman7@gmail.com

Mahasakti Mahamaya

mahamaya.2008@gmail.com

1 Civil Engineering Department, National Institute of Technology Rourkela, Rourkela, Odisha 769008, India
Keywords Stabilized earth - Cement stabilization . Prediction model · Artificial intelligence $\cdot$ Functional network $\cdot$ Multivariate adaptive regression spline

\section{Introduction}

Population surge in the recent decades has led to rapid industrialization and urbanization, which has increased the demand for massive infrastructures. However, since the land with adequate bearing capacity is limited, hence, the shift is graduating towards land with soft soil, even though they are not suitable for building infrastructure. These type of soil are characterized by high plasticity, higher fraction of fines and high void ratio, with low strength and high compressibility [1]. There is a requirement of land reclamation to have increased strength and density, reduced compressibility and permeability to solve stability, settlement, ground water, and other environmental-related problems to build infrastructure in these areas.

In situ stabilization of soil using chemical and physical means are the most widely used methods for the land reclamation. Chemical stabilization, particularly cement stabilization is more prefered method for in situ stabilization of soft soil, due to its quick gain of strength and economy. Many researchers have shed light on the mechanism of strength improvement in soft soil using cement stabilization techniques. The effects of water content, cement content, curing time, and compaction energy on the engineering characteristics of cement-stabilized soils have been emphasized [1-13].

The present practice is to perform laboratory tests on soft clays admixed with cementing agents to determine the cement contents required for a given application. A predictive equation which can accurately determine the gain in 
strength of the cement-soil mix will not only save a lot of time but will also lead to an optimum and effective utilization of the cement.

Kaniraj and Havanagi [14] conducted unconfined compression tests on samples of cement stabilized fly ash-soil mixture compacted at optimum moisture content with cement content varying from 3 to $9 \%$. Based on the results, a prediction model for the unconfined compressive strength with respect to cement content, fly ash content and curing period was given. Miura et al. [7] identified the water cement ratio as the critical factors governing the engineering behaviour of high water content cement stabilized clay and presented a model equation for unconfined compressive strength as a function of water cement ratio. An empirical relationship based on cement content, water content and curing period was proposed to predict the strength development in cement-soil mix by Tan et al. [2]. Based on Abram's law, Horpibulsuk et al. [10] developed the normalized empirical model to predict the strength of cement stabilized soil using the clay-water cement ratio as the principal parameter. Horpibulsuk et al. [12] through experiments on cement admixed soft clays concluded that clay-water/cement ratio is a microstructural parameter and proposed a model equation to calculate the cement content required to reach a desired strength. The prediction model take care of any variations in the clay-water ratio during the stabilization process by allowing the change in cement content during the stabilization process.

Artificial intelligence (AI) techniques are considered as alternate statistical method by many researchers and are found to be better in prediction as compared to the empirical methods [15-19]. Narendra et al. [20] carried out laboratory experiments on cement stabilized red earth $(\mathrm{CL})$, brown earth $(\mathrm{CH})$ and black cotton soil $(\mathrm{CH})$ and presented a predictive model for unconfined compressive strength using genetic programming (GP). The developed model contained liquid limit, liquidity index, water content, cement content, curing period, $\mathrm{pH}$ and sodium ion concentrations $(\mathrm{Na}+)$ as the inputs. However, the output was found to be more dependent on the water cement ratio, cement content and curing period rather than the soil properties, as only three types of soil samples were considered. Alavi et al. [21] studied application of artificial neural network (ANN) to predict maximum dry density (MDD) and unconfined compressive strength (UCS) of stabilized soil and ANN was found to be a better prediction technique. Das et al. [22] used ANN and support vector machine (SVM) to predict the MDD and UCS of cement stabilized soil based on soil plasticity (LL, PI), clay content $(C)$, sand content $(S)$, gravel content $(G)$, moisture content (MC) and cement content (CC) as inputs. Based upon different statistical performances criteria such as correlation coefficient (R), absolute average error (AAE), maximum absolute error (MAE), root mean square error (RMSE) and overfitting ratio (OR), SVM was found to be a better prediction technique than ANN.

However, the ANN has poor generalization, attributed to attainment of local minima during training and needs iterative learning steps to obtain better learning performances. The SVM has better generalization compared to ANN, but the parameters $(C)$ and insensitive loss function ( $\varepsilon$ ) need to be fine-tuned by the user. Moreover, these techniques will not produce a comprehensive model equation and are also called as "black box" system [23]. Therefore, the present study is an attempt to develop comprehensive model equations to determine the MDD and UCS of cement stabilized soil with the use of two recently developed AI techniques; functional networks (FN) and multivariate adaptive regression splines (MARS). Multilinear regression (MLR) model was also developed to compare the results.

\section{Methodology}

As the use of FN [24, 25], and MARS [25-27] is limited in geotechnical, these are described briefly in the following sections.

\section{Functional Networks}

ANNs have been extensively used in all the fields of science and engineering including geotechnical engineering and have been proved to be an able prediction tool. The FN introduced by Castillo [28, 29], Castillo and Ruiz-Cobo [30] and Castillo et al. [31, 32] is a powerful extension of the ANNs and is advantageous to ANNs. Owing to their advantages over ANNs, FNs have been successfully used in various fields such as petroleum engineering [33], signal processing, pattern recognition, function's approximations [34] real-time flood forecasting, science, bioinformatics, medicine [35] structural engineering [36], transportation engineering [37] and geotechnical engineering [24, 25].

In FN the selection of neuron functions is a two-step process. In the first step, called as the structural learning, the functions are selected and in the second step, known as the parametric learning the functions are estimated. The second step is same as the determination of weights by the ANNs. The FN can use arbitrary multiargument and vector valued functions, whereas in ANNs can use only fixed (sigmoid) functions.

Figure 1 shows the essential elements of a FN. As is evident from the Fig. 1, each network contains storing units to store the inputs $\left(x_{1}, x_{2}, x_{3}\right)$, outputs $\left(f_{4}, f_{5}\right)$ and intermediate information $\left(X_{4}, X_{5}\right)$ and directed links to connect the various input, output and intermediate units in 


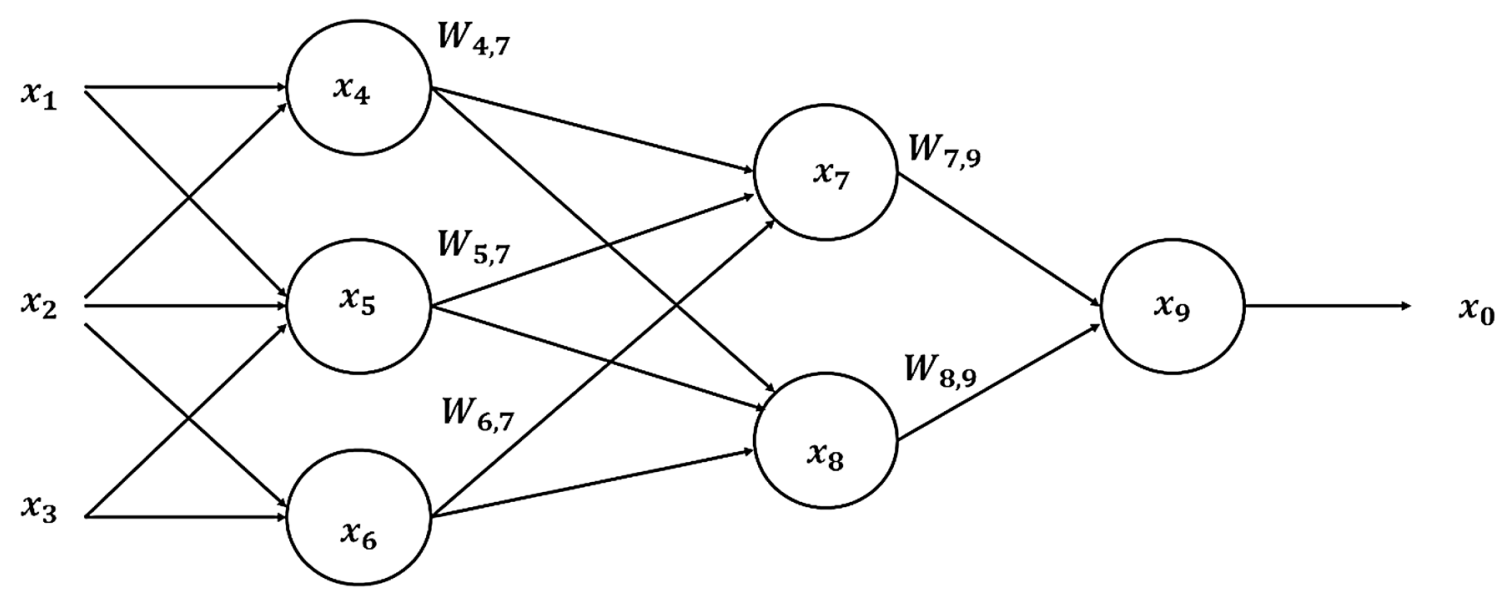

Fig. 1 A functional network showing its essential components

accordance with the advance of the FN algorithm. Based on physical relationship between input and output the initial topology of the FN is selected according to the properties of the data available. For a given topology, a unique neuron function is arrived that produces a set of output. Then the network is trained with available data minimizing the Euclidean norm of the error function, given by

$E=\frac{1}{2} \sum_{i=1}^{n}\left(O_{i}-F(i)\right)^{2}$

The $O_{i}$ is the observed value of the output and the approximate neural function $f_{i}(x)$ may be arranged as

$f_{i}(x)=\sum_{j=1}^{m} a_{i j} \emptyset_{i j}(x)$

where $\emptyset=$ shape functions with algebraic expressions $\left(1, x, x^{2}, x^{3} \ldots x^{n}\right)$, trigonometric functions such as $[1, \sin (x), \cos (x), \sin (2 x), \cos (2 x), \sin (3 x), \cos (3 x)], \quad$ or exponential functions such as $\left(1, e^{x}, e^{2 x}, \ldots, e^{n x}\right)$. The associative optimization function may lead to a system of linear or nonlinear algebraic equations. This paper applies the associativity FNs. In general, with the use of the basic theory of functional equations, any multi-input network can be transformed to an associative network [30, 32]. Further details regarding the algorithm for associative FN can be found in Castillo [28].

\section{Multivariate Adaptive Regression Splines}

Multivariate adaptive regression spline (MARS) is an adaptive regression technique, popularized by Friedman [38] for solving regression-type problems. MARS is a 'white box' technique [23] as it is based on physical laws and underlying physical relationships of the system can be explained. The MARS algorithm does not presume any relationship between the dependent and independent variables. It builds a relationship between the inputs and the output using a set of coefficients and basis function (BF)s, which are determined entirely from the data set for the particular problem. This makes MARS particularly useful for problems involving more number of inputs.

Divide and conquer strategy is the backbone of MARS algorithm. It divides the training data set into a number of piecewise linear segments called splines of different gradients. The end points of splines are known as knots and the piece-wise linear functions or piece-wise cubic functions between two knots is known as a BF. For the present study, only piece-wise linear functions have been used in this study.

The MARS model is of the form of equation:

$f(x)=\beta_{0}+\sum_{i=1}^{M} \beta_{m} \lambda_{m}(x)$

where each $\lambda_{\mathrm{m}}$ is a BF, which can be a spline or a product of two or more splines. The coefficients $\beta$ are constants estimated using the least squares method. A BF is of the form $\max (0, x-t)$ where $t$ is the location of a knot. It is defined as,

$\max (0, x-t)=\{x-t, \quad$ if $x>t$ or, 0 otherwise $\}$

The MARS algorithm consists of two step process to fit the data; forward step and backward pruning process. In forward step process starting with the intercept $\beta_{0}$, BFs are added in each subsequent step to reduce the errors in training step. This may lead to an overfitted model. In second step, backward pruning process, generalized crossvalidation (GCV) technique is used to avoid the overfitting. The GCV penalizes both BFs and knots, thereby reducing overfitting or improving generalization of the model.

For a data with $N$ samples, GCV is calculated using the equation: 
$\mathrm{GCV}=\frac{\frac{1}{N} \sum_{i=1}^{N}\left[Y_{i}-f\left(X_{i}\right)\right]^{2}}{\left[1-\frac{M+d(M-1) / 2}{N}\right]^{2}}$

where $M$ is the number of BFs, $d$ is the penalizing parameter, $N$ is the number of data sets and $f\left(X_{i}\right)$ denotes the predicted values of the MARS model. The denominator of GCV is responsible for increasing variance in case of increasing model complexity. The term $(M-1) / 2$ in the denominator represents the number of knots. Thus, GCV penalizes BFs as well as knots.

\section{Database and Preprocessing}

A database available in literature [39] and used by [22] has been considered for developing the prediction models in this study. The inputs in the data set consist of liquid limit (LL), plastic limit (PI) (\%), percentage of sand (S), percentage of gravel $(\mathrm{G})$, moisture content $(\mathrm{MC})$ and the cement content (CC). The MDD (in $\mathrm{kN} / \mathrm{m}^{3}$ ) and UCS (in $\mathrm{N} / \mathrm{m}^{2}$ ) were taken as the outputs for building the MDD and the UCS prediction models, respectively. The maximum, minimum, average and standard deviation values of the parameters used in the present study is shown in Table 1 .

The data set for building MDD model consist of 58 samples out of which 42 randomly selected data were taken for training the models and 16 were used for testing. The data set for building UCS model consisted of 51 samples out of which 37 randomly selected data were taken for training the models and the rest 14 were used for testing. The data was normalized between 0 and 1 before using it for the FN and MARS analysis. The FN and MARS [40] algorithm were implemented using Matlab [41].

\section{Results and Discussion}

The development of the FN and MARS models, the prediction equations and the comparison of FN and MARS model with the multi linear regression (MLR) analysis and previously available ANN and SVM model are presented as follows.

\section{FN Model}

Associative FN model with five inputs and one output, as shown in Fig. 2, were developed for the prediction of MDD and UCS in this study. The complexity and accuracy of a FN model depends upon the nature and degree of the BF chosen. An increase in the degree of the chosen BF generally leads to more accurate model but at the same time it leads to a more complex model equation. So, a trade off was made between the complexity and accuracy while adopting a model for the present problem. The corresponding model equation is given by

$y=a_{0}+a_{i j} \sum_{i=1}^{n} \sum_{j=1}^{m} f_{i}\left(x_{j}\right)$

where $n$ is the number of inputs in the $\mathrm{FN}$ model and $\mathrm{m}$ is the degree of the basis function used. In the present study, cosine function was chosen as the basis function. The value of $a_{0}$ and the coefficients $a_{i j}$ are determined from the FN training algorithm.

The best model for the MDD and UCS prediction were obtained with degree 5 and 4, respectively. The corresponding model equations for the MDD and UCS are given by Eqs. (7) and (8), respectively.

$\operatorname{MDD}\left(\right.$ in $\left.\frac{\mathrm{kN}}{\mathrm{m}^{3}}\right)=-96267.2+a_{i j} \sum_{i=1}^{n} \sum_{j=1}^{m} f_{i}\left(x_{j}\right)$

Table 1 Parameters of data considered for present study

\begin{tabular}{|c|c|c|c|c|c|c|c|c|}
\hline Model & L.L. (\%) & P.I. (\%) & Clay (\%) & Sand $(\%)$ & Gravel (\%) & Moisture content $(\%)$ & Cement content $(\%)$ & $\mathrm{DD}\left(\mathrm{kN} / \mathrm{m}^{3}\right)$ \\
\hline \multicolumn{9}{|l|}{ MDD } \\
\hline Min & 18.00 & 0.00 & 6.00 & 34.00 & 0.00 & 6.90 & 3.00 & 14.91 \\
\hline $\operatorname{Max}$ & 48.00 & 48.00 & 53.00 & 94.00 & 46.00 & 19.00 & 6.00 & 21.68 \\
\hline Avg & 32.23 & 12.21 & 23.95 & 64.50 & 11.66 & 9.96 & 5.59 & 18.80 \\
\hline \multirow[t]{2}{*}{ SD } & 11.23 & 9.69 & 10.20 & 15.55 & 13.89 & 2.77 & 0.73 & 1.66 \\
\hline & & & & & & & & UCS $\left(\mathrm{N} / \mathrm{mm}^{2}\right)$ \\
\hline \multicolumn{9}{|l|}{ UCS } \\
\hline Min & 18.00 & & 0.00 & 6.00 & 34.00 & 6.90 & 3.00 & 1.10 \\
\hline Max & 73.00 & & 48.00 & 53.00 & 94.00 & 46.00 & 6.00 & 4.70 \\
\hline Avg & 31.55 & & 12.32 & 24.16 & 62.48 & 13.44 & 5.54 & 2.76 \\
\hline
\end{tabular}




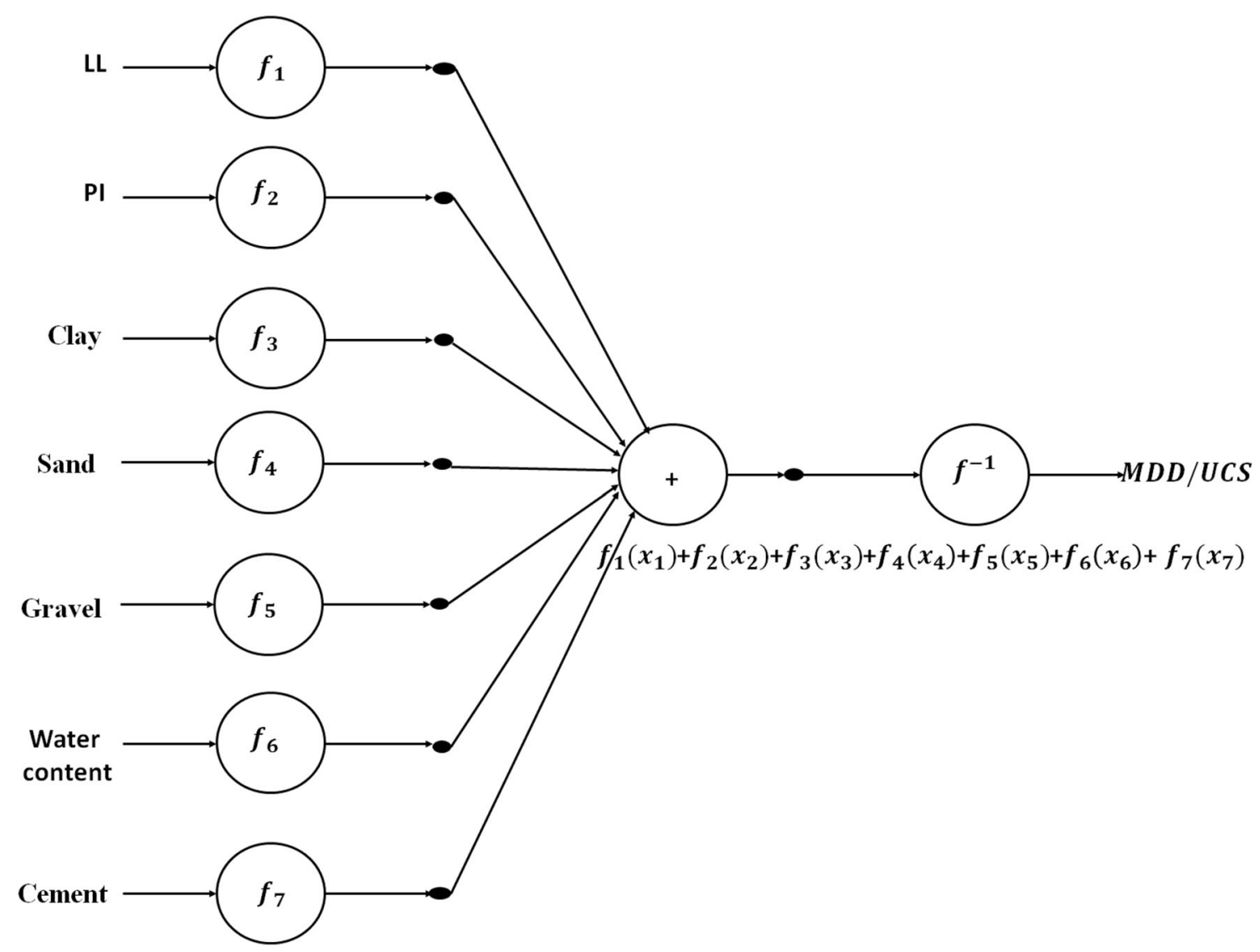

Fig. 2 Associativity functional network used in this study

$$
\begin{aligned}
f_{1}\left(x_{j}\right)= & -1.00281 \times \operatorname{cosLL} \\
f_{2}\left(x_{j}\right)= & 9.9353 \times \cos \mathrm{PI}-2.2882 \times \cos 2 \mathrm{PI} \\
f_{3}\left(x_{j}\right)= & 35024.4 \times \cos C-21428 \times \cos 2 C+9019.174 \\
& \times \cos 3 C-2366.08 \times \cos 4 C+295.443 \times \cos 5 C \\
f_{4}\left(x_{j}\right)= & 1.8737 \times \cos 2 S-1.8634 \times \cos 3 S \\
f_{5}\left(x_{j}\right)= & 17665.04 \times \cos G-10866.88 \times \cos 2 G \\
& +4611.12 \times \cos 3 G-1220.68 \times \cos 4 G \\
& +153.2106 \times \cos 5 G \\
f_{6}\left(x_{j}\right)= & 111413.0106 \times \cos \mathrm{MC}-68071.3239 \times \cos 2 \mathrm{MC} \\
& +28542.0363 \times \cos 3 \mathrm{MC}-7417.0681 \\
& \times \cos 4 \mathrm{MC}+907.4254 \times \cos 5 \mathrm{MC}
\end{aligned}
$$

$f_{7}\left(x_{j}\right)=0.0995 \times \cos 3 \mathrm{CC}$

$$
\mathrm{UCS}\left(\text { in } \frac{\mathrm{N}}{\mathrm{m}^{2}}\right)=3078.2131+a_{i j} \sum_{i=1}^{n} \sum_{j=1}^{m} f_{i}\left(x_{j}\right)
$$

$$
\begin{aligned}
f_{1}\left(x_{j}\right)= & 35207.3382 \times \cos \mathrm{LL}-18360.7562 \times \cos 2 \mathrm{LL} \\
& +5631.7652 \times \cos 3 \mathrm{LL}-778.9098 \times \cos 4 \mathrm{LL}
\end{aligned}
$$

$$
\begin{aligned}
f_{2}\left(x_{j}\right)= & -45424.7841 \times \cos \mathrm{PI}+23901.5767 \times \cos 2 \mathrm{PI} \\
& -7438.6540 \times \cos 3 \mathrm{PI}+1049.2719 \times \cos 4 \mathrm{PI}
\end{aligned}
$$

$$
\begin{aligned}
f_{3}\left(x_{j}\right)= & 9.3312 \times \cos C-4.2603 \times \cos 2 C \\
f_{4}\left(x_{j}\right)= & 3055.2498 \times \cos S-1694.0927 \times \cos 2 S \\
& +576.2671 \times \cos 3 S-93.5490 \times \cos 4 S
\end{aligned}
$$

$$
f_{5}\left(x_{j}\right)=1834.6819 \times \cos G-1012.4206 \times \cos 2 G
$$$$
+340.6439 \times \cos 3 G-54.4633 \times \cos 4 G
$$

$f_{6}\left(x_{j}\right)=274.6706 \times \cos \mathrm{MC}-124.9777 \times \cos 2 \mathrm{MC}$ $+25.5542 \times \cos 3 \mathrm{MC}$

$f_{7}\left(x_{j}\right)=0.1450 \times \cos \mathrm{CC}$

The inputs to be entered in Eqs. (7) and (8) are the normalized value of the inputs between 0 and 1. Equations (8) 
and (9) give the normalized value of the MDD and UCS respectively between 0 and 1 . The actual values of MDD and UCS can be calculated using the Eqs. (9) and (10), respectively.

$$
\begin{aligned}
& \mathrm{MDD}_{\text {actual }}=6.77 \times \mathrm{MDD}_{\text {norm }}+14.91 \\
& \mathrm{UCS}_{\text {actual }}=3.6 \times \mathrm{UCS}_{\text {norm }}+1.1
\end{aligned}
$$

Figures 3 and 4 give the scatter plot between the measured and predicted values of MDD and UCS, respectively. It can be seen from the Figs. 3 and 4 that scatter of data from the line of equality is within $80 \%$ prediction limit for both MDD and UCS. The correlation coefficient $(R)$ value between the measured and the predicted valued of the MDD was found to be 0.908 and 0.922 for the training and testing data set, respectively. The corresponding values for the UCS prediction were 0.927 and 0.951 . These values suggest a strong correlation $(|R|>0.8)$ in accordance to Smith [42]. However, it is also known that $R$ is a biased estimate [43], hence the efficiency of the developed models was also considered according to Nash-Sutcliff coefficient of efficiency $(E)$ [44]. The $E$ values for training and testing data set for the MDD and UCS model are provided in Table 4. The generalization of model is measured in terms of the overfitting ratio (OR), which is the ratio of RMSE of the testing data to that of training data. The OR close to 1 shows a good generalization by the model. The OR for the MDD and UCS models are found out to be 1.13 and 0.976 , respectively showing good generalization of the developed model.

\section{MARS Model}

The complexity and accuracy of a MARS model depends upon the maximum number of BFs allowed in the final

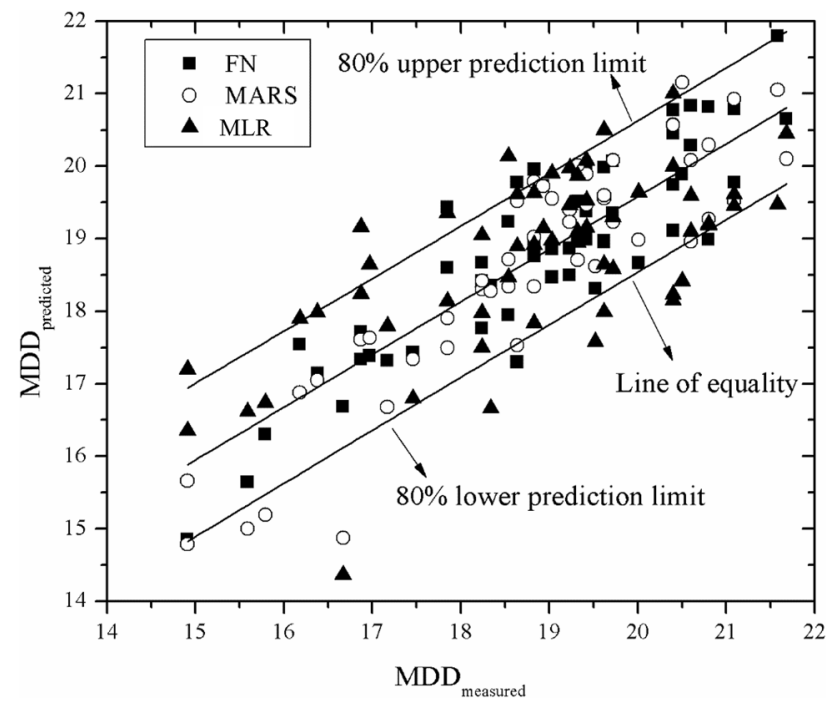

Fig. 3 Scatter plot between the measured and predicted values of MDD according to FN, MARS and MLR modelling

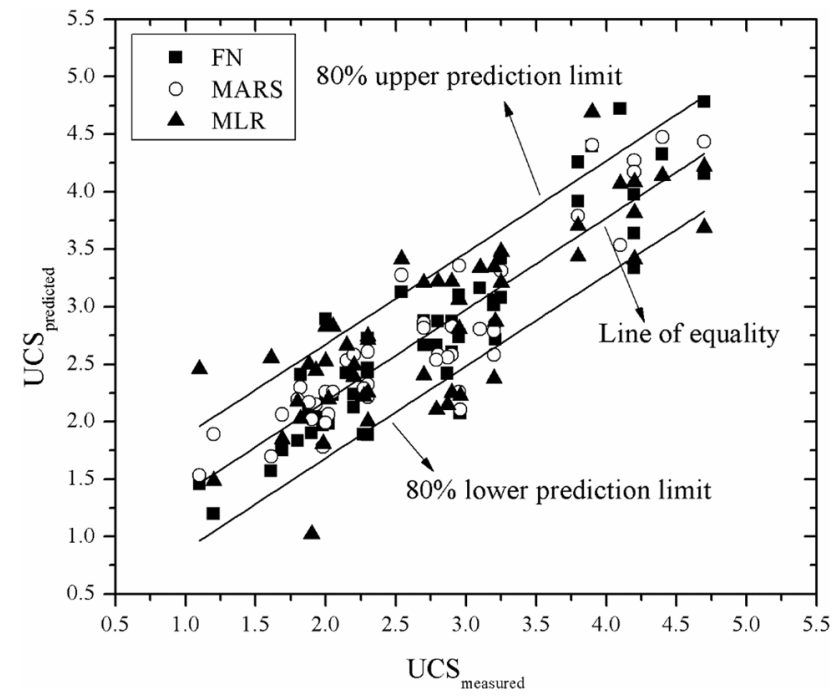

Fig. 4 Scatter plot between the measured and predicted values of UCS according to FN, MARS and MLR modelling

result. If more number of BFs are allowed, the model achieved will be more accurate but at the same time, it will be complex the model equation will be lengthy. Hence, a tradeoff needs to be made between accuracy and complexity in this case also. MARS models with 9 BFs were adopted in this study to develop prediction models for MDD and UCS. The corresponding equation for the model for each parameter is provided in Eqs. (11) and (12) respectively.

$$
\begin{aligned}
\operatorname{MDD}\left(\operatorname{in} \frac{\mathrm{kN}}{\mathrm{m}^{3}}\right)= & 0.6360-1.2631 \times \mathrm{BF} 1-3.0761 \\
& \times \mathrm{BF} 2+8.6173 \times \mathrm{BF} 3-21.7470 \\
& \times \mathrm{BF} 4-28.5876 \times \mathrm{BF} 5-3.8010 \\
& \times \mathrm{BF} 6+1.1885 \times \mathrm{BF} 7+2.7682 \times \mathrm{BF} 8 \\
& +0.3274 \times \mathrm{BF} 9
\end{aligned}
$$

The BFs corresponding to Eq. (11) are provided in Table 2.

$$
\begin{aligned}
\mathrm{UCS}\left(\operatorname{in} \frac{\mathrm{N}}{\mathrm{m}^{2}}\right)= & 0.1914+1.2208 \times \mathrm{BF} 1+11.8112 \\
& \times \mathrm{BF} 2+3.5029 \times \mathrm{BF} 3-63.3180 \\
& \times \mathrm{BF} 4-1.0002 \times \mathrm{BF} 5+114.6534 \\
& \times \mathrm{BF} 6+3.3040 \times \mathrm{BF} 7-794.9950 \\
& \times \mathrm{BF} 8-147.5978 \times \mathrm{BF} 9
\end{aligned}
$$

The BFs corresponding to prediction model for UCS (Eq. 12) are provided in Table 3.

Similar to FN models, the inputs to be entered in Eqs. (11) and (12) are the normalized value of the inputs between 0 and 1. Equations (11) and (12) give the normalized value of the MDD and UCS respectively between 
Table 2 BFs corresponding to MDD prediction model using MARS

\begin{tabular}{llll}
\hline Basis function & Expression & Basis function & Expression \\
\hline BF1 & $\max (0, \mathrm{MC}-0.0909)$ & $\mathrm{BF} 6$ & $\mathrm{BF} 1 \times \max (0,0.7167-\mathrm{S})$ \\
$\mathrm{BF} 2$ & $\max (0,0.0909-\mathrm{MC})$ & $\mathrm{BF} 7$ & $\max (0,0.2766-\mathrm{C})$ \\
BF3 & $\mathrm{BF} 1 \times \max (0, \mathrm{C}-0.2766)$ & $\mathrm{BF} 8$ & $\max (0, \mathrm{C}-0.2766) \times \max (0,0.2174-\mathrm{G})$ \\
BF4 & $\mathrm{BF} 3 \times \max (0, \mathrm{PI}-0.2917)$ & $\mathrm{BF} 9$ & $\max (0, \mathrm{G}-0.0870)$ \\
BF5 & $\mathrm{BF} 3 \times \max (0,0.2917-\mathrm{PI})$ & & \\
\hline
\end{tabular}

Table 3 BFs corresponding to UCS prediction model using MARS

\begin{tabular}{llll}
\hline Basis function & Expression & Basis function & Expression \\
\hline BF1 & $\max (0,0.3542-\mathrm{PI})$ & $\mathrm{BF} 6$ & $\max (0, \mathrm{~S}-0.6167) \times \max (0, \mathrm{C}-0.3617)$ \\
$\mathrm{BF} 2$ & $\mathrm{BF} 1 \times \max (0,0.2979-\mathrm{C})$ & $\mathrm{BF} 7$ & $\mathrm{BF} 1 \times \max (0, \mathrm{C}-0.2609)$ \\
$\mathrm{BF} 3$ & $\max (0,0.6167-\mathrm{S}) \times \max (0, \mathrm{PI}-$ & $\mathrm{BF} 8$ & $\mathrm{BF} 2 \times \max (0, \mathrm{MC}-0.1240)$ \\
& $0.1042)$ & & $\max (0,0.6167-\mathrm{S}) \times \max (0,0.1042-\mathrm{PI}) \times \max (0, \mathrm{MC}-$ \\
$\mathrm{BF} 4$ & $\max (0,0.6167-\mathrm{S}) \times \max (0, \mathrm{~S}-0.5167)$ & $\mathrm{BF} 9$ & $0.1488)$ \\
& $\max (0,0.6167-\mathrm{S}) \times \max (0,0.5167-\mathrm{S})$ & & \\
\hline
\end{tabular}

0 and 1. The actual values of MDD and UCS can be calculated using the Eqs. (9) and (10), respectively. It may be mentioned here that the above explicit model equation for both MDD and UCS as per FN and MARS model can be calculated in a programmable calculator instead of going to intrinsic part of MARS or FN.

Figures 3 and 4 give the scatter plot between the measured and predicted values of MDD and UCS according to MARS model along with the results of FN model. For the MARS model also, most of the data lie within the $80 \%$ prediction limit for both MDD and UCS. The $R$ value between the measured and the predicted values of the MDD for the MARS model was found to be 0.925 and 0.922 for the training and testing data set, respectively. The corresponding values for the UCS prediction were 0.927 and 0.954 . These values also suggest a strong correlation in accordance with Smith [42]. The $R$ values for MDD according to MARS model are marginally better than those of FN model. However, the $R$ values for UCS according to both MARS and FN model are close. The $\mathrm{E}$ values for training and testing data set for the MDD model are 0.856 and 0.644 , respectively. The corresponding values for UCS model are 0.851 and 0.814 , respectively. The $E$ value for MDD in training is better for MARS than that for FN. The trend is reverse for the testing data set. For the UCS model, the $E$ value for training data set according to the MARS and FN model are close. However, the $\mathrm{E}$ value for the testing data set was found to be lower for the MARS model than the FN model. The OR for the MDD and UCS models according to MARS were found out to be 1.61 and 0.91 , respectively. The high value of OR value for MDD shows a poor generalization. As per Das and Basudhar [44], efficiency of a model should be compared in terms of testing data rather than training data. Hence, based on the comparison in terms of $R$ and $E$ for testing data and OR values FN model was found to be better than MARS model for prediction of MDD. But, the MARS prediction model for UCS was found to be better than the FN model.

\section{Multilinear Regression Model (MLR Model)}

Using the above data, a MLR model was also developed and is presented as follows for comparison with AI models.

The prediction equations for MDD and UCS according to MLR analysis are presented in Eqs. (13) and (14).

$$
\begin{aligned}
\operatorname{MDD}\left(\text { in } \frac{\mathrm{kN}}{\mathrm{m}^{3}}\right)= & 33.9-0.0064 \times \mathrm{LL}+0.0523 \times \mathrm{PI} \\
& -0.03 \times C-0.064 \times S-0.073 \times G \\
& -0.482 \times \mathrm{MC}-0.924 \times \mathrm{CC} \\
\mathrm{UCS}\left(\text { in } \frac{\mathrm{N}}{\mathrm{m}^{2}}\right)= & -17.1-0.0155 \times \mathrm{LL}-0.0305 \times \mathrm{PI} \\
& +0.203 \times C+0.228 \times S+0.248 \times G \\
& -0.0223 \times \mathrm{MC}-0.268 \times \mathrm{CC}
\end{aligned}
$$

Figures 3 and 4 give the scatter plot between the measured and predicted values of MDD and UCS according to MLR model also. For the MLR model, some of the data lie outside the $80 \%$ prediction limit showing a poorer prediction for the MLR model as compared to the FN and MARS models. The $R$ and $E$ values for the MLR model for the MDD and UCS models are also shown in Table 4. 
Though OR value as per MLR model the MDD and UCS models are found to be 1.22 and 1.12 , respectively, showing good generalization but prediction model is not efficient as $R(0.75$ and 0.73$)$ and $E(0.21$ and 0.35$)$ values for both MDD and UCS models, particularly for testing data are very less.

\section{Comparison with Other AI Techniques}

The data set used in this study has been used by Das et al. [22] to predict MDD and UCS using ANN and SVM. Das et al. [22] have used different ANN models named as Bayesian regularization neural network (BRNN), Levenberg-Maqruardt neural network (LMNN) and differential evolution neural network (DENN). The comparison of the MAE, AAE and RMSE is presented in Figs. 5, 6, 7, 8. For MDD model, Figs. 5 and 6 present the MAE, AAE and RMSE for the training and testing data set, respectively. Similarly for the UCS model, Figs. 7 and 8 present the MAE, AAE and RMSE for the training and testing data set, respectively. Table 4 compares the values of $R, E$ and overfitting ratio (OR) for the prediction techniques used by Das et al. [22] and those used in this study. It can be seen from the Table 4 than both FN and MARS are better than the ANN models for MDD prediction in terms of different statistical criteria. Only the MAE in training was found to be higher for the FN and MARS model than different ANN models. Though, performance of the SVM in terms of $R$ and MAE, AAE and RMSE in training for MDD prediction was found better than the FN and MARS model. The performance of SVM is same as that of FN and MARS for the testing data set. Hence, it can be inferred that FN and MARS are a better tool than ANN and SVM for prediction of MDD.

A similar trend was observed between the FN, MARS and ANN models for the UCS prediction also, i.e., FN and MARS outperformed thee ANN models. The $R$ value for the SVM model for UCS prediction in training was found to better than those of FN and MARS, but the values of MAE, AAE and RMSE were found to be lower for the FN and MARS models. The FN and MARS techniques have a better prediction performance than the SVM technique for the testing data set for UCS prediction. Thus, FN and MARS prove to be a better prediction tool than ANN and SVM for prediction of UCS also. FN, MARS, ANN and SVM outperformed the MLR model for the MDD and the UCS prediction. However, FN model is better than MARS model in terms of generalization i.e. lower OR values.

\section{Sensitivity Analysis}

The sensitivity analysis is carried out for a model to find out the relative importance of input parameters in affecting the output. Since, the MARS model did not incorporate all the inputs and the MLR model was found to have a poor performance, the sensitivity analysis according to $\mathrm{FN}$ model only has been presented in this study. The sensitivity analysis was carried out according to Gandomi et al. [45]. To calculate the sensitivity of a given input, only the values of the said input were varied whereas the value of all other inputs was kept constant equal to their average value. The sensitivity was calculated according to the Eqs. (15) and (16).

$N_{i}=f_{\max }\left(x_{i}\right)-f_{\min }\left(x_{i}\right)$

$S_{i}=N_{i} / \sum_{i=1}^{n} N_{i}$

where $f_{\max }\left(x_{i}\right)$ and $f_{\min }\left(x_{i}\right)$ are the maximum and minimum of the predicted output over the $i$ th input domain respectively, when the other inputs are equal to their average values, $n$ is the number of variables which is equal to 7 in the present study. Table 5 shows the sensitivity analysis of inputs for FN model for both MDD and UCS. The moisture content (MC) and specific gravity $(G)$ are found to be the most important inputs for the MDD and UCS models, respectively. Identification of $\mathrm{MC}$ as the most important
Table 4 Comparison of R, E and OR for the MDD and UCS prediction using ANN, SVM, FN, MARS and MLR

\begin{tabular}{|c|c|c|c|c|c|c|c|c|c|c|}
\hline & \multicolumn{5}{|c|}{ MDD } & \multicolumn{5}{|l|}{ UCS } \\
\hline & \multicolumn{2}{|c|}{ Training } & \multicolumn{2}{|c|}{ Testing } & \multirow[t]{2}{*}{ OR } & \multicolumn{2}{|c|}{ Training } & \multicolumn{2}{|c|}{ Testing } & \multirow[t]{2}{*}{ OR } \\
\hline & $\mathrm{R}$ & $\mathrm{E}$ & $\mathrm{R}$ & $\mathrm{E}$ & & $\mathrm{R}$ & $\mathrm{E}$ & $\mathrm{R}$ & $\mathrm{E}$ & \\
\hline BRNN & 0.91 & 0.81 & 0.84 & 0.69 & 1.79 & 0.86 & 0.81 & 0.87 & 0.69 & 1.80 \\
\hline DENN & 0.91 & 0.81 & 0.88 & 0.73 & 1.67 & 0.88 & 0.81 & 0.85 & 0.73 & 1.66 \\
\hline LMNN & 0.81 & 0.64 & 0.76 & 0.53 & 1.59 & 0.86 & 0.64 & 0.84 & 0.53 & 1.58 \\
\hline SVM & 0.95 & & 0.93 & & 1.63 & 0.98 & & 0.92 & & \\
\hline FN & 0.91 & 0.82 & 0.92 & 0.73 & 1.13 & 0.93 & 0.86 & 0.95 & 0.70 & 0.98 \\
\hline MARS & 0.92 & 0.86 & 0.94 & 0.64 & 1.61 & 0.92 & 0.85 & 0.95 & 0.81 & 0.91 \\
\hline MLR & 0.67 & 0.45 & 0.75 & 0.21 & 1.22 & 0.81 & 0.65 & 0.73 & 0.35 & 1.12 \\
\hline
\end{tabular}


Fig. 5 Bar chart showing MAE, AAE and RMSE for the training data set in MDD according to ANN, SVM, FN, MARS and MLR analysis

Fig. 6 Bar chart showing MAE, AAE and RMSE for the testing data set in MDD according to ANN, SVM, FN, MARS and MLR analysis

Fig. 7 Bar chart showing MAE, AAE and RMSE for the training data set in UCS according to ANN, SVM, FN, MARS and MLR analysis

\section{MAE AAE 国 RMSE}

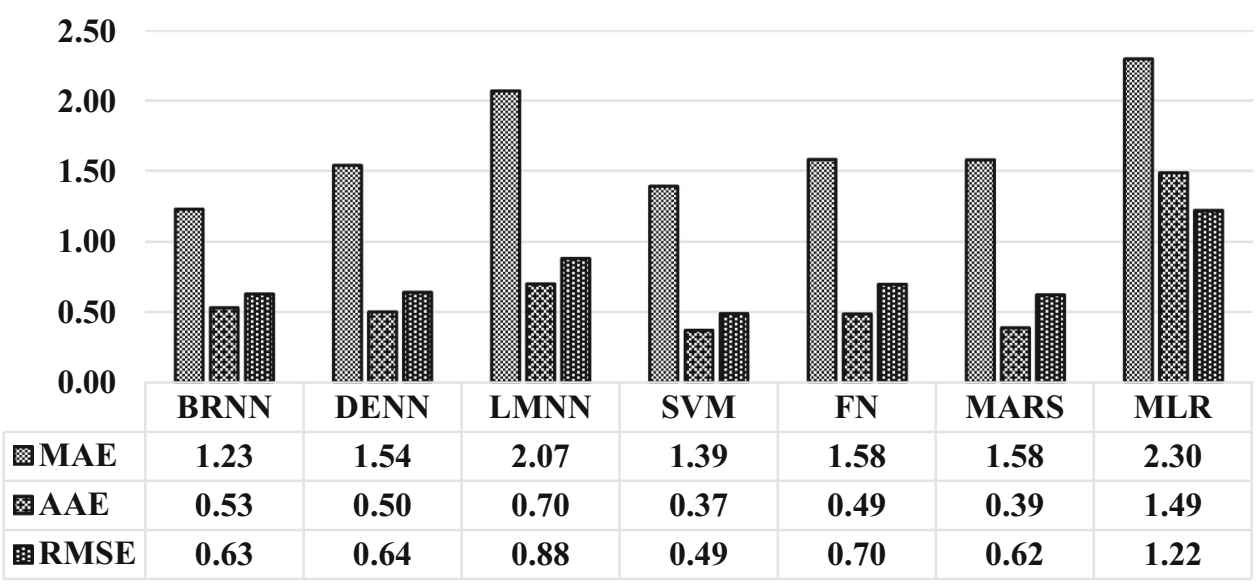

\section{MAE AAE MMSE}

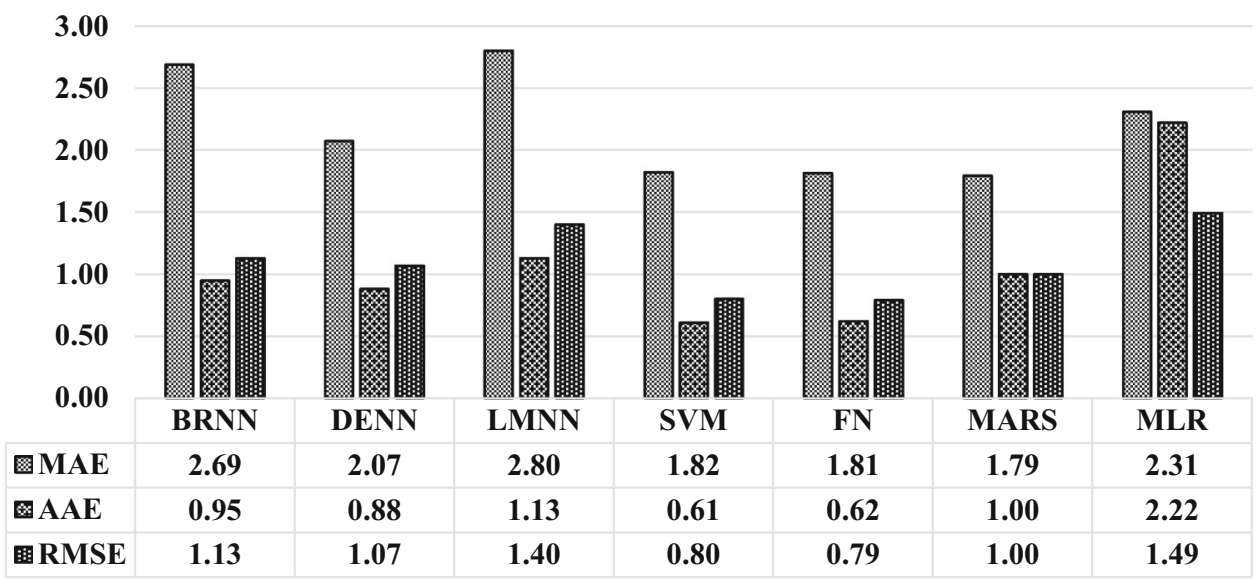

\section{MAE AAE 四 RMSE}

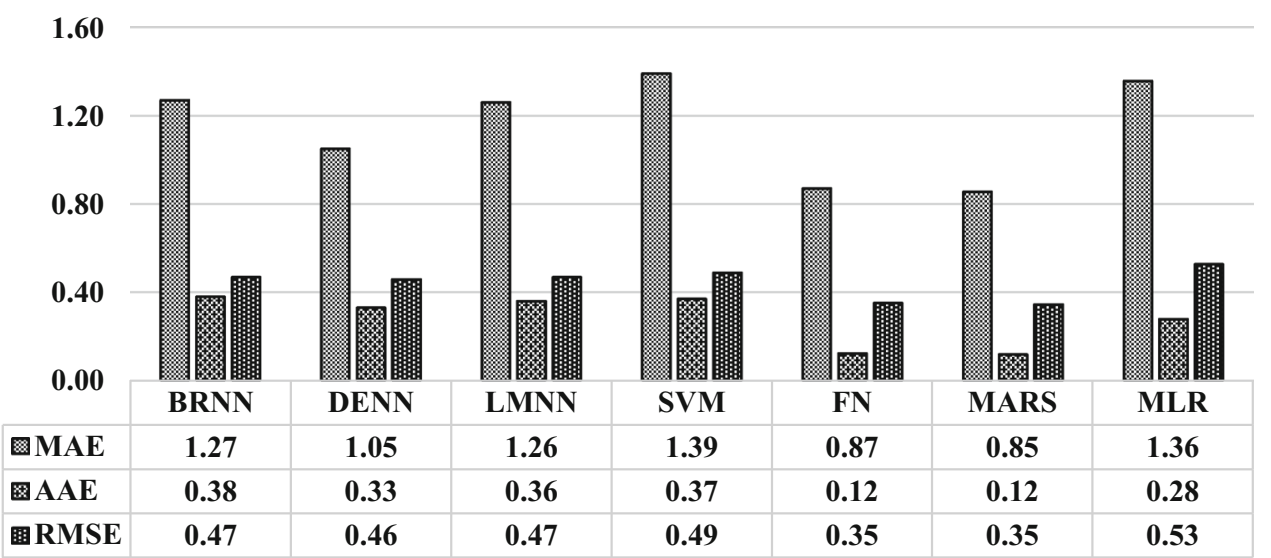

parameter for the MDD model was found to be in accordance with Das et al. [22]. The cement content is found to be the least important input both for the MDD and the UCS models. This may suggest that for low cement stabilization it is more important to have proper grading of the mixture than the cement content. 
Fig. 8 Bar chart showing MAE, AAE and RMSE for the testing data set in UCS according to ANN, SVM, FN, MARS and MLR analysis
MAE 圆 AAE 圆 RMSE

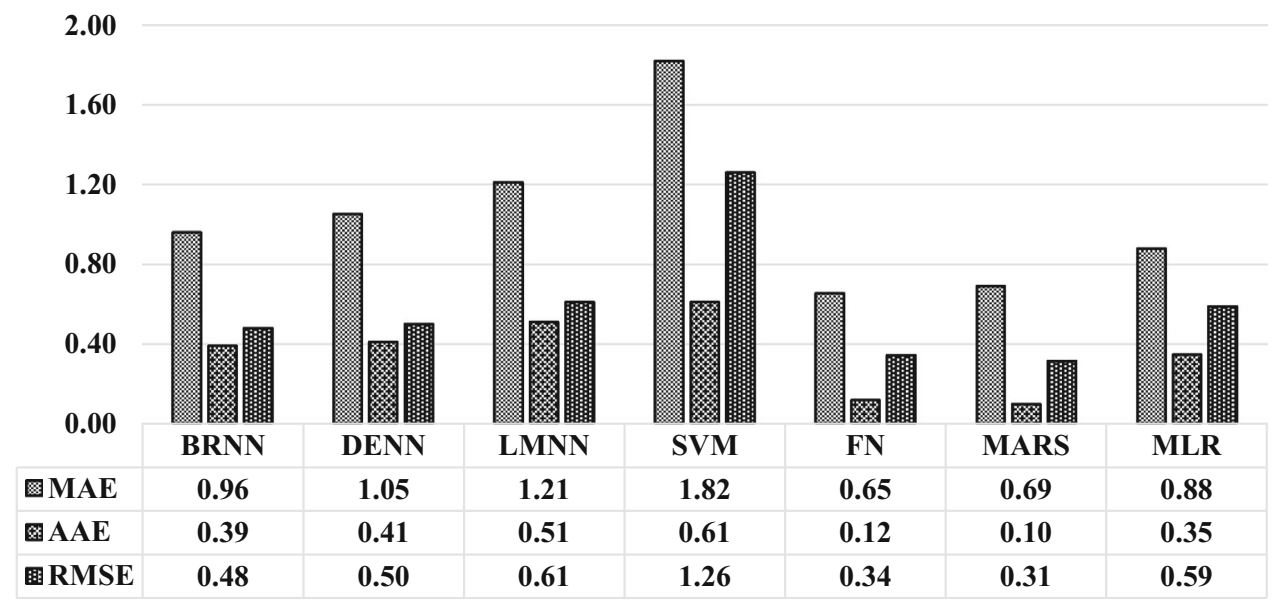

Table 5 Sensitivity analysis of inputs for the MDD and UCS models according to FN using Gandomi et al. (2013)

\begin{tabular}{lll}
\hline Inputs & Rank & \\
\cline { 2 - 3 } & MDD model & UCS model \\
\hline LL & 6 & 3 \\
PI & 5 & 4 \\
C & 2 & 5 \\
S & 4 & 2 \\
G & 3 & 1 \\
MC & 1 & 6 \\
CC & 7 & 7 \\
\hline
\end{tabular}

\section{Conclusions}

In the present study an attempt has been made to develop prediction models for both the MDD and UCS of cement stabilized soil using two recently developed AI techniques; FN and MARS.

Based on different statistical criteria like correlation coefficient, coefficient of efficiency, maximum absolute error and root mean square error for testing (new) data, FN and MARS prediction models for both MDD and UCS are found to be better than existing ANN and SVM models. Based on overfitting ratio, it was also observed that FN and MARS have better generalization than the ANN and SVM models. In terms of $R$ and $E$ for testing data and OR values FN model was found to be better than MARS model for prediction of MDD. But, the MARS prediction model for UCS was found to be better than the FN model. Sensitivity analysis shows that $\mathrm{MC}$ and $G$ are the most important parameters for the MDD and UCS prediction respectively. The cement content was found to be the least important parameter for both MDD and UCS prediction. This may suggest that for low cement stabilization, it is more important to have proper grading/packing of materials than the cement content. Unlike ANN and SVM, the prediction models for both the MDD and UCS based on FN and MARS are very comprehensive and can be used by the professional engineers for the initial trial of different mixture.

\section{References}

1. Porbaha A (1998) State of the art in deep mixing technology: Part I. Basic concepts and overview. In: Proceedings of the ICEground improvement vol 2, No. 2, pp 81-92

2. Tan TS, Goh TL, Yong KY (2002) Properties of Singapore marine clays improved by cement mixing. ASTM Geotech Test J 25(4):422-433

3. Uddin K, Balasubramaniam AS, Bergado DT (1997) Engineering behavior of cement treated Bangkok soft clay. Geotech Eng J 28(1):89-119

4. Yin JH, Lai CK (1998) Strength and stiffness of Hong Kong marine deposit mixed with cement. Geotech Eng J 29(1):29-44

5. Consoli NC, Rotta GV, Prietto PDM (2000) Influence of curing under stress on the triaxial response of cemented soil. Geotechnique 50(1):99-105

6. Kasama K, Ochiai H, Yasufuku N (2000) On the stress-strain behaviour of lightly cemented clay based on an extended critical state concept. Soils Found 40(5):37-47

7. Miura N, Horpibulsuk S, Nagaraj TS (2001) Engineering behavior of cement stabilized clay at high water content. Soils Found 41(5):33-45

8. Horpibulsuk S, Miura N (2001) A new approach for studying behavior of cement stabilized clays. In: Proceedings of the 15th international conference on soil mechanics and geotechnical engineering (ISSMGE), Istanbul, Turkey, pp 1759-1762

9. Horpibulsuk S, Miura N, Nagaraj TS (2003) Assessment of strength development in cement-admixed high water content clays with Abrams' law as a basis. Geotechnique 53(4): 439-444 
10. Horpibulsuk S, Bergado DT, Lorenzo GA (2004) Compressibility of cement admixed clays at high water content. Geotechnique 54(2):151-154

11. Horpibulsuk S, Miura N, Bergado DT (2004) Undrained shear behavior of cement admixed clay at high water content. J Geotech Geoenviron Eng ASCE 30(10):1096-1105

12. Horpibulsuk S, Miura N, Nagaraj TS (2005) Clay-water/cement ratio identity of cement admixed soft clay. J Geotech Geoenviron Eng ASCE 131(2):187-192

13. Horpibulsuk S, Katkan W, Sirilerdwattana W, Rachan R (2006) Strength development in cement stabilized low plasticity and coarse grained soils: laboratory and field study. Soils Found 46(3):351-366

14. Kaniraj SR, Havanagi VG (1999) Compressive strength of cement stabilized fly ash-soil mixtures. Cem Concr Res 29(5):673-677

15. Das SK, Basudhar PK (2006) Undrained lateral load capacity of piles in clay using artificial neural network. Comput Geotech 33:454-459

16. Das SK, Biswal RK, Sivakugan N, Das B (2011) Classification of slopes and prediction of factor of safety using differential evolution neural networks. Environ Earth Sci 64(1):201-210

17. Muduli PK, Das MR, Samui P, Das SK (2013) Uplift capacity of suction caisson in clay using artificial intelligence techniques. Mar Georesour Geotechnol 31(4):375-390

18. Hoanga ND, Phamb AD (2016) Hybrid artificial intelligence approach based on meta heuristic and machine learning for slope stability assessment: a multinational data analysis. Expert Syst Appl 46:60-68

19. Shahin MA (2016) State-of-the-art review of some artificial intelligence applications in pile foundations. Geosci Front 7:33-44

20. Narendra BS, Sivapullaiah PV, Suresh S, Omkar SN (2006) Prediction of unconfined compressive strength of soft grounds using computational intelligence techniques: a comparative study. Comput Geotech 33(3):196-208

21. Alavi AH, Gandomi AH, Mollahassani A, Heshmati AA, Rashed A (2010) Modeling of maximum dry density and optimum moisture content of stabilized soil using artificial neural networks. J Plant Nutr Soil Sci 173(3):368-379

22. Das SK, Samui P, Sabat AK (2011) Application of artificial intelligence to maximum dry density and unconfined compressive strength of cement stabilized soil. Geotech Geol Eng 29(3):329-342

23. Giustolisi O, Doglioni A, Savic DA, Webb BW (2007) A multimodel approach to analysis of environmental phenomena. Environ Model Softw 22(5):674-682

24. Khan SZ, Suman S, Pavani M, Das SK (2015) Prediction of the residual strength of clay using functional networks. Geosci Front. doi:10.1016/j.gsf.2014.12.008

25. Das SK, Suman S (2015) Prediction of lateral load capacity of pile in clay using multivariate adaptive regression spline and functional network. Arab J Sci Eng 40(6):1565-1578. doi:10. 1007/s13369-015-1624-y

26. Samui P, Das S, Kim D (2011) Uplift capacity of suction caisson in clay using multivariate adaptive regression spline. Ocean Eng 38(17-18):2123-2127
27. Samui P, Kim D, Viswanathan R (2015) Spatial variability of rock depth using adaptive neuro-fuzzy inference system (ANFIS) and multivariate adaptive regression spline (MARS). Environ Earth Sci 73(8):4265-4272. doi:10.1007/s12665-014-3711-x

28. Castillo E (1998) Functional networks. Neural Process Lett 7:151-159

29. Castillo E, Cobo A, Gomez-Nesterkin R, Hadi AS (2000) A general framework for functional networks. Networks 35(1):70-82

30. Castillo E, Ruiz-Cobo R (1992) Functional equations and modelling in science and engineering. Marcel Dekker, New York

31. Castillo E, Cobo A, Gutierrez JM, Pruneda E (1998) An introduction to functional networks with applications. Kluwer, Boston

32. Castillo E, Cobo A, Manuel J, Gutierrez JM, Pruneda E (2000) Functional networks: a new network-based methodology. Comput Aided Civ Infrastruct Eng 15:90-106

33. El-Sebakhy EA, Asparouhov O, Abdulraheem A, Al-Majed A, Wu D, Latinski K, Raharja I (2012) Functional networks as a new data mining predictive paradigm to predict permeability in a carbonate reservoir. Expert Syst Appl 39(12):10359-10375

34. Castillo E, Cobo A, Gutierrez JM, Pruneda RE (1999) Working with differential, functional and difference equations using functional networks. Appl Math Model 23:89-107

35. El-Sebakhy EA, Faisal KA, Helmy T, Azzedin F, Al-Suhaim A (2006) Evaluation of breast cancer tumor classification with unconstrained functional networks classifier. In: Proceeding of the 4th international conference on computer systems and applications, Sharjah, pp 281-287

36. Rajasekaran S (2004) Functional networks in structural engineering. J Comput Civ Eng 18:172-181

37. Attoh-Okine NO (2005) Modeling incremental pavement roughness using functional network. Can J Civ Eng 32:899-905

38. Friedman J (1991) Multivariate adaptive regression splines. Ann Stat 19:1-141

39. Burroughs VS (2001) Quantitative criteria for the selection and stabilization of soils for rammed earth wall construction. Ph.D. Thesis submitted to University of New South Wales

40. Jekabsons G (2011) ARESLab: Adaptive Regression Splines toolbox for Matlab/Octave. http://www.cs.rtu.lv/jekabsons/. Accessed 10 Oct 2014

41. Math Work Inc (2005) Matlab User's Manual, Version 6.5. Natick (MA)

42. Smith GN (1986) Probability and statistics in civil engineering: an introduction. Collins, London

43. Das SK, Sivakugan N (2010) Discussion of intelligent computing for modeling axial capacity of pile foundations. Can Geotech J 47:928-930. doi:10.1139/T10-048

44. Das SK, Basudhar PK (2008) Prediction of residual friction angle of clays using artificial neural network. Eng Geol 100(3-4):142-145

45. Gandomi AH, Yun GJ, Alavi AH (2013) An evolutionary approach for modeling of shear strength of RC deep beams. Mater Struct 46(12):2109-2119 